\title{
Energy Management System to Prevent Blackout in Smart Grid Network - A Case Study of Kotoka International Airport
}

\author{
Emmanuel Nartey ${ }^{*}$, Isaac Owusu-Nyarko \\ Department of Electrical/Electronic, Regional Maritime University, Accra, Ghana
}

Email address:

nattyembongo@hotmail.com (E. Nartey)

*Corresponding author

\section{To cite this article:}

Emmanuel Nartey, Isaac Owusu-Nyarko. Energy Management System to Prevent Blackout in Smart Grid Network - A Case Study of Kotoka International Airport. Journal of Electrical and Electronic Engineering. Vol. 4, No. 4, 2016, pp. 83-88. doi: 10.11648/j.jeee.20160404.11

Received: August 1, 2016; Accepted: August 11, 2016; Published: September 7, 2016

\begin{abstract}
Efforts to tackle energy deficit have become a global challenge. Not a day passes by without global media outlets publishing on the subject of energy management and this has drawn public attention since it is not only help users to monitor and control their power consumption easily, but also reduce their electricity bill. Ghana Airport Company has been facing a challenge of paying high electricity tariffs as a result of the non-aeronautical service providers at the airport. This has cause revenue lost as a result of misuse of certain equipment's in the various departments in the company. Various scheduling models have been proposed to optimize power consumption. However, they are few solutions to prevent the blackout while optimizing power consumption. We design and implement Energy Management system (EMS) which can autonomously control the power consumption below a given threshold while negotiating electricity consumption with the smart grid system. By keeping the power consumption below a given threshold autonomously, the power demand will not exceed the available supply, therefore can prevent the blackout. Since the threshold can be negotiated with the smart grid system, company satisfaction can be increased.
\end{abstract}

Keywords: Ghana Airport Company, Energy Management, Blackout, Electricity Tariffs

\section{Introduction}

The Ghana Airports Company Limited (GACL) was established as a result of the decoupling of the existing Ghana Civil Aviation Authority (GCAA) in line with the modern trends in the aviation industry. The company was registered in January 2006 with the responsibility for planning, developing, managing and maintaining all airports and aerodromes in Ghana namely Kotoka International Airport (KIA) and the regional airports namely Kumasi, Tamale, Sunyani as well as various air trips. The Company commenced business on 1st January 2007. The long queues and congestion that characterized activities at the airport especially during peak seasons are now confined to history. The phased project, which began in 2014, has seen the expansion of the existing floor area of the arrival hall by $5,148 \mathrm{~m}^{2}$, provision and installation of two new baggage handling equipment to bring to four the number of baggage carousels in the arrival hall, Provision and installation of twenty-six immigration booths an increase of 14 over the existing 12 booths, replacement of air-condition systems. Other completed projects include the installation of state-ofthe-art security systems with ultra-modern IP based real time cameras with video analytics to provide 24 hour surveillance for both airside and landside to aid in capturing and deterring criminal activities at the airport. The Phase 3 Development project is also ongoing and includes the reconstruction of taxiway, rehabilitation and extension of fuel mains and the provision of aeronautical ground lighting (AGL). For airport operators around the world, the highest priority is always the safety of passengers and employees. And this safety simply cannot be ensured without a reliable supply of electrical power for the control of Ghana Civil Aviation Authority equipment's. It's vital for the smooth interoperation of the various complex technologies for monitoring and controlling air traffic, for handling passengers and baggage, and for building automation including the use of installed security systems. And because speed is essential in the event of a fault or a dangerous situation, airport operators need a reliable and 
fast overview of the components in the power network and efficient access to their controls. This is the only way to ensure that safe operation is restored in the shortest possible time. The power supply for an airport usually comprises switching and distribution systems for the high, medium and low-voltage levels, complete with local automation and protection systems. It may also include power generation plants and the required emergency power systems ensuring smooth operation at all times require an energy automation system tailored to the individual subsystems. This guarantees that there is always sufficient electrical power available wherever and whenever it is needed. The increasing complexity of these subsystems creates a wide variety and high density of information, and airport operators require a master control system to effectively and productively use this data. Airport Company has been facing a challenge of paying high electricity tariffs as a result of the non-aeronautical service providers at the airport. This has led the company to lose revenue as the use of approved gadgets in the various offices is on the rise. It is on this backdrop that we want to design and construct a load management system to be able to trip the system when a preset amount of energy assigned to a particular unit is exceeded. This is an attempt to regulate the use of energy at the airport so that the critical equipment can be in use all day for the efficient and effective of the airport. Even though the entire energy management plan may not be able to produce the magnitude of savings produced by the project, the remaining projects in the pipeline have the potential of meeting the airport's energy targets by 2020 .

\section{Smart Grid}

Smart grids use computation and communication to improve the efficiency and dependability of power generation, transmission, and distribution. As such, they are among the most critical and complex cyber-physical systems. The rising level of developments in generation and consumption of power have put lots of stress on distribution networks. These developments have brought cyber control on distribution network with more efficient use of the limited capacity available. However, each additional component used in this cyber control is capable of causing a failure, and the net effect of this increased vulnerability and complexity on the overall reliability of the grid requires proper examination. Every comprehensive reliability model should be able to consider every potential source as a failure and such failure will have overall effect on the entire power system. The main categories of components that comprise a smart grid have been briefly explained as shown below;

\subsection{Physical Infrastructure Components}

Electric transmission systems are primarily composed of current-carrying components, including generators and transmission lines. The classification of the physical infrastructure into hierarchical levels where generation, transmission, and distribution facilities form levels also determines reliability of the entire power system. However, in the complex electric power grid, Distributed Energy Resources (DERs) are considered generation facilities that are dispersed throughout the electricity network [25].

\subsection{Control Devices}

The control of power flow has traditionally relied on generator control and phase-shifting and tap-changing voltage regulation transformers. In addition, both series reactors and series capacitors are also used to control power flow. The recent technological development in electrical power systems has introduced the use of FACTS components. The FACTS concept is the use of power electronic devices in the high-voltage side of the network, to make it electronically controllable. The Unified Power Flow Controller (UPFC), the Static Compensator (STATCOM), and the Interphase Power Controller (IPC) are examples of FACTS components.

\subsection{Communication}

The integration of high-speed and reliable data communication networks to manage the complex power grid effectively and intelligently is an important feature of smart grids [25]. Information exchange among distributed power devices to assist the functioning of management systems is being done by the communication backbone of power systems. The reliability of power management depends on a reliable communication backbone. In other words, power systems cannot operate correctly unless reliable communication takes place among intelligent electronic devices [24]. Communication networks used in smart grids are typically of three types: wide-area networks, field area networks, and home area networks.

\section{Energy Management}

Energy management is a broader term, which applies differently in different scenarios, but we are concerned about the one which is related with energy saving in homes, public sector/ government organizations or business. In this scenario the process of monitoring, controlling and conserving energy in an organization/ building may be termed as energy management [1]. In smart grid where the consumers can generate local energy from several distributive generation units and where there is a plenty of space for different pricing schemes, the need for energy management programs has been pointed out by many researchers. In [2, 3] Wireless Sensor Networks (WSNs) have been used for an in-home energy management (iHEM) application. The schemes are based on communication between smart appliances, a central Energy Management Unit (EMU), smart meter and storage unit. The algorithms applied have been designed for ToU pricing scheme, where electricity charges rates are different for different periods of time. iHEM application manages the home energy by shifting the load form peak to off-peak periods i.e. reduces peak load demand. When a consumer turns on an appliance a data packet is sent to the EMU. EMU 
then communicates with smart meter and local generation units to know about the price information from utility and the available local energy respectively. Based on this information, EMU schedules the starting time of the appliance. Waiting time of the appliance is calculated as the difference between the suggested time by EMU and request start time. Demand response today is envisioned as a method to reduce the costs of electric energy by discouraging customers from using electric energy from the grid during high demand periods. These high demand periods carry disproportionate costs for the power grid largely due to the requisite generation and transmission capacity needed to meet this demand [4]. The highest 100 hours of the year are estimated to contribute to between ten and twenty percent of electricity costs [38]. Demand-side energy management can help defray these costs. Perhaps the most well-known demand-side energy management technique is peak shaving. The objective of peak shaving is to reduce the peak power demand by controlling deferrable loads in the system [5]. Peak shaving has been demonstrated to reduce system costs by as much as twenty five percent [40]. Utilities have reduced peak period costs by implementing demand response programs, including real time pricing, time-of-use pricing, and critical peak-pricing programs [6]. Full customer participation in such demand response pro electric power demand is a result of load management and reduction, distributed generation, distributed energy storage, development and implementation of more efficient technologies and public awareness. Advances in information technology are expanding the potential usefulness of demand response [7] by creating the opportunity to turn the power grid into a Smart Grid [8]. As the communication of prices approaches real time, loads will be able to more precisely optimize energy use, generation, and storage [9-10] according to customer preference [11-12]. A method developed in [13] for aggregating demand response data could be used to more accurately predict customer response to operational real time changes in price. Using a near real time pricing signal, the utility could potentially control the load profile [14], increase system security [15], and stabilize grid frequency [16] and more [17-18]. This level of control will require an advanced communication infrastructure [19] and will likely impact utility planning [20], grid operations [21] and energy markets [22-23]. An alternative to a pricedriven demand response program is direct control of loads through a demand dispatch control signal [24]. However, even a direct control over loads can use price as a control signal. Since the amount of electric energy demand is dependent on price, the calculation of the pricing signal is central to the operation of a demand response driven Smart Grid.

\section{System Design}

This section has been divided into three main parts. The first covers the selection of components with justification. The second part talks about the design specifications of the components selected and the software design for the system.
The final section talks about the construction of the system and testing.

\subsection{Components Selection}

\subsubsection{Arduino Mega 1280 Microcontroller}

The Arduino Mega was selected for this project based on the following considerations:

- Excellent Performance: The arduino mega board is capable of providing faster processing speed needed to perform the task which include signal output.

- Another consideratiom made when selection Arduino Mega for this project is that it is an open source.

\subsubsection{Gateway}

A gateway is a hardware device that acts as a "gate" between two networks. It may be a router, firewall, server, or other device that enables traffic to flow in and out of the network. While a gateway protects the nodes within network it is also a node itself. The gateway node is considered to be on the "edge" of the network as all data must flow through it before coming in or going out of the network. It may also translate data received from outside networks into a format or protocol recognized by devices within the internal network.

\subsubsection{Switch}

A switch is used to network multiple computers together. Switches made for the consumer market are typically small, flat boxes with 4 to 8 Ethernet ports. These ports can connect to computers, cable or DSL modems, and other switches. High-end switches can have more than 50 ports and often are rack mounted.

\subsubsection{Relays}

A Relay is a device that completes a connection between two contact points by means of a mechanical movement. A relay may use a solenoid to magnetically move a contact switch. A signal is sent to the relay which can be used as the source of the electromagnet. They are typically used as switching mechanisms or serves as isolation, allowing for small DC current to switch large AC or DC currents.

\subsection{Components Specification-Arduino}

Table 1. Arduino Technical Specification.

\begin{tabular}{ll}
\hline Operating Votage & $5 \mathrm{~V}$ \\
Input Voltage (recommended) & $7-12 \mathrm{~V}$ \\
Digital I/O pin & 54 \\
Analog input & 16 \\
DC current for 3.3V pin & $50 \mathrm{~mA}$ \\
Flash memory & $128 \mathrm{~KB}$ \\
RAM & $8 \mathrm{~KB}$ \\
EEPROM & $4 \mathrm{~KB}$ \\
Clock Speed & $16 \mathrm{MHz}$ \\
Manufacturer & Atmel \\
\hline
\end{tabular}


Arduino Uno Ethernet Shield Specification

- Requires an Arduino board (not included)

- Operating voltage 5V (supplied from the Arduino Board)

- Ethernet Controller: W5100 with internal 16K buffer

- Connection speed: $10 / 100 \mathrm{Mb}$

\subsection{Software Design}

The program has been divided into two main parts. The void setup section which describes the speed rate at which the Arduino is communicating with the computer and the speed rate of the communication. In this section, the program must run once during the start of the program. The other section is the void loop which runs the program continuously in the loop forever.

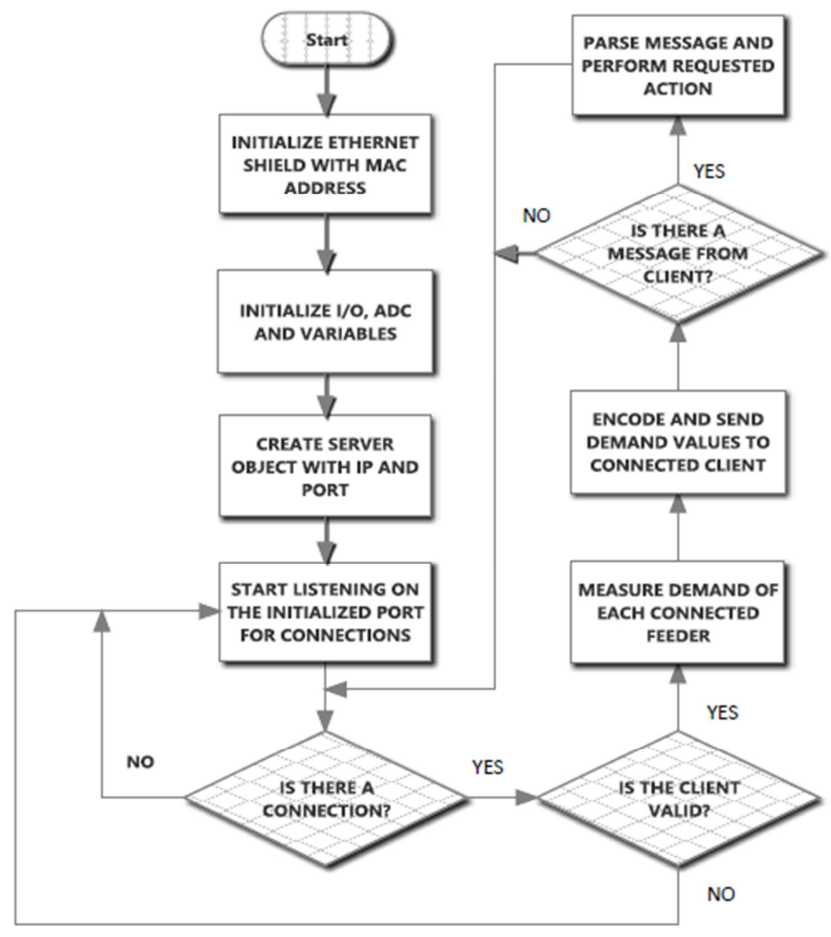

Figure 1. System Flow Chart.

\section{System Construction}

This section describes the wiring of the components which consists of, Controller, Relays, Arduino Ethernet shield, LED bulbs, and a Computer.

Layout steps for Hardware Construction

- The Arduino Uno Ethernet shield was slotted into the microcontroller

- The controller then was connected to the three (3) way relay

- The relay output is connected to the three (3) individual loads basically filament bulbs

- The set-up was then mounted on a wooden board.

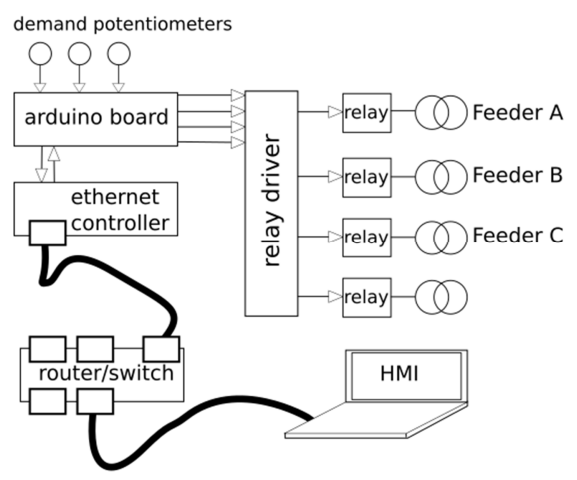

Figure 2a. Block Diagram of the System.

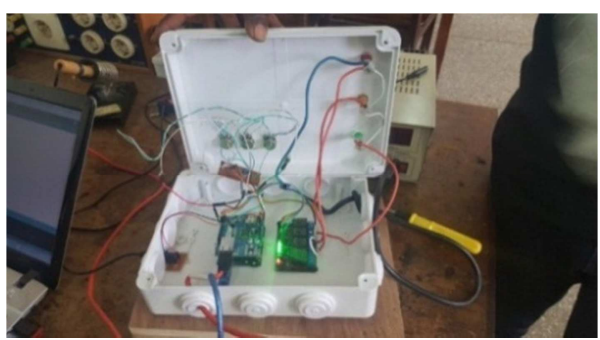

Figure 2b. Snap shot of System Construction

\section{System Testing and Result}

The graphs below represent the results of load management system for Kotoka International Airport (K.I.A). The system consists of a control server, the gateway and a set of appliances. The control server collects all the information from the gateway, save and calculate the power consumption, which is represented with a graph. Gateway is connected with some controllable appliances and sends them to the server. When the control server receives available power supply data, it sets a threshold for power consumption and sends the threshold to the gateway. The appliances are divided into several priority levels according to the importance and a customer's requirement. This project also shows that the priority level can be decided manually by users since each user may have different preferences.

There are three feeders labelled Red for TERMINAL 1, Black for TERMINAL 2 Blue for HEARDQUARTERS with maximum consumption power of $15.31 \mathrm{KW}, 14.65 \mathrm{KW}$ and $12.25 \mathrm{KW}$ respectively. Figure 3 shows the graph of normal power consumption of loads on the feeders.

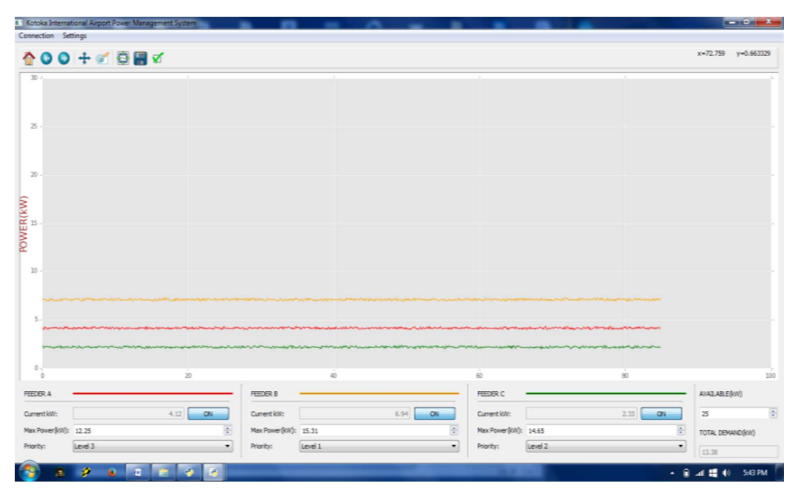

Figure 3. Loads operating within allocated power demand. 


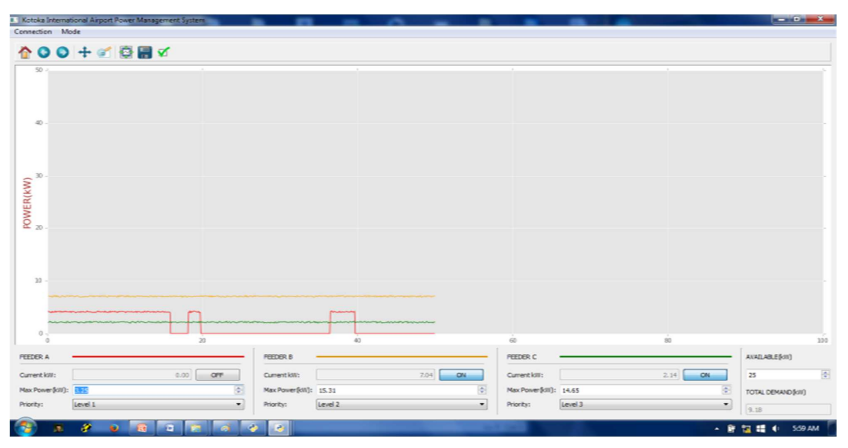

Figure 4. When feeder A exceeds the allocated power.

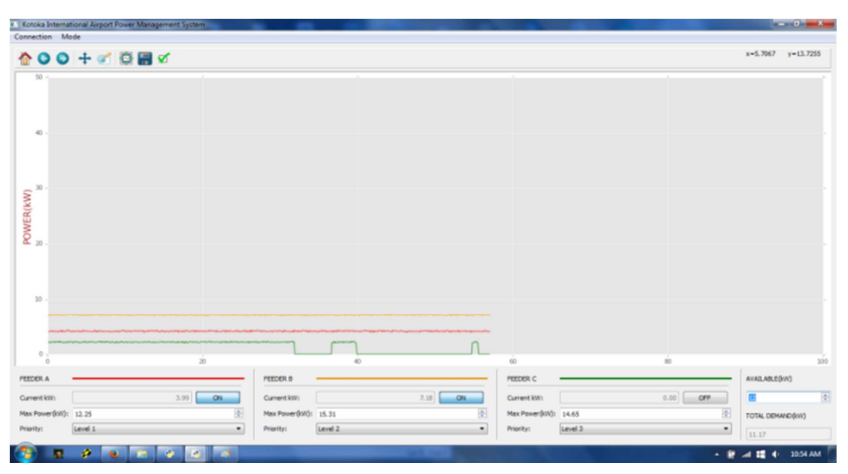

Figure 5. When the available power is reduced.

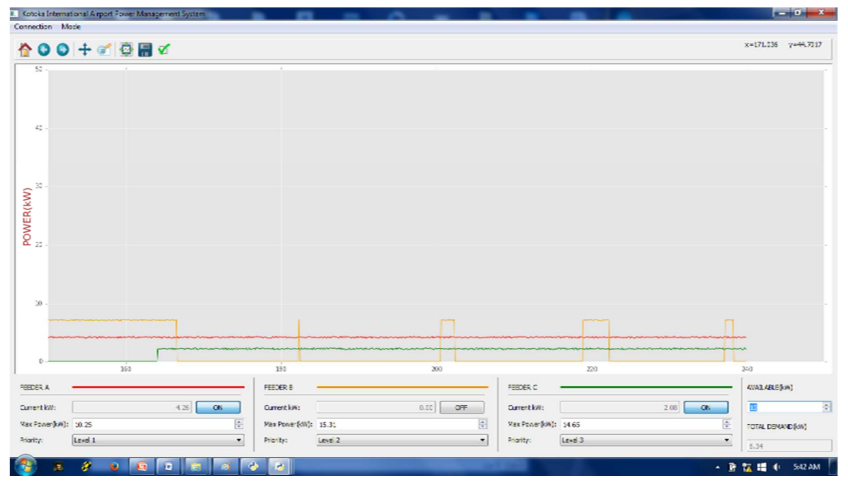

Figure 6. Further reduction in available power.

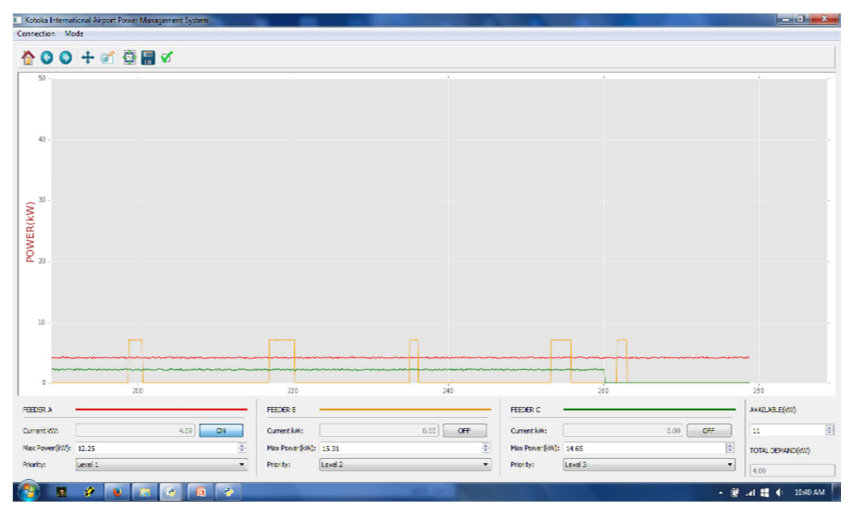

Figure 7. Manual shutting down of the feeders.

\section{Discussion}

This project is aimed at giving Kotoka International Airport (K.I.A) a Load Management System (LMS) that is Smart grid based, which provides monitoring and controlling individual electrical loads, $t$ improving efficiency and save cost on energy bills.

Investigations of the electrical power consumption of K.I.A reveals that load usage is higher at Terminal 1 where the airline offices are situated, followed by Terminal 2 and the least is the Headquarters, but the priority level during the day should be Terminal 1 and headquarters in that order of power consumption, the evening priority level should be, Terminal 2 where we have the Customer User Terminal

\section{Equipment.}

The design and implementation provides a load management system that allows electrical loads to operate within an allocated power demand and a system that is able to trip off when the load demand on the line exceeds the allocated power provided.

Under normal conditions as in figure 3, the graph shows linear output and individual load lines are parallel to each other. When a load line exceeds the allocated power demand, it drops and thereby shutting down the load line as showed in figure 4.

\section{Conclusion}

Smart-grid metering and control systems hold enormous promise for improving efficiency, convenience, and sustainability. Smart Grid Load Management System (SGLMS) at Kotoka International Airport (K. I. A) will be the most effective way to ensure sustainability, efficiency and provide pragmatic growth as it will enable the institution to make the right and informed decisions concerning its consumption of energy and saving cost on its energy bills.

An implementation of energy management system scheduling model has been proposed to prevent blackout while optimizing the available power consumption. Threshold negotiation depending on user requirement provides a solution to fulfil the power requirement when there is not enough energy and or regulate energy consumption, by keeping the power usage below the threshold line; the system can prevent the blackout while optimizing the usage of the available power.

Electricity consumption awareness is a basic step to save energy in various offices and buildings. This modern technology will be used for balancing demand side load management. Consumers can also be motivated to change their behavior for energy saving. The proposed system distributes and balances the consumer load all over the day. The functions make it possible by setting the priority of the appliances to participate in load management that automatically reduces peak load.

\section{References}

[1] M. Erol-Kantarci and H. T. Mouftah, "Wireless sensor networks for cost-efficient residential energy management in the smart grid," Smart Grid, IEEE Transactions on, vol. 2, no. 2, pp. 314-325, 2011. 
[2] . Shao, S.; Pipattanasomporn, M.; Rahman, S. Development of Physical-Based Demand Response-Enabled Residential Load Models. IEEE Trans. Power Syst. 2013, 28, 607-614.

[3] G. W. Arnold, "Challenges and Opportunities in Smart Grid: a Position Article," Proceedings of the IEEE, vol. 99, no. 6, pp. 922-927, 2011.

[4] J. Wells, "Electricity Markets-Consumers Could Benefit From Demand Programs, But Challenges Remain," Government Accountability Office, Washington, DC, 2004.

[5] C. F. Jack, "Peak Shaving - a Way to Fight Rising Costs," IEEE Transactions on Industry Applications, vol. IA 12, no. 5, pp. 486-491, September 1976.

[6] J. Malinowski and K. Kaderly, "Peak Shaving - a Method to Reduce Utility Costs," in Region 5 Conference: Annual Technical and Leadership Workshop, April 2004.

[7] Federal Energy Regulatory Commission, "Assessment of Demand Response and Advanced Metering," Staff Report, February 2011. http://www.ferc.gov/legal/staff-reports/06-09demand-response.pdf. [Accessed 4 June 2013].

[8] Peter Palensky and Dietmar Dietrich, "Demand Side Management: Demand Response, Intelligent Energy Systems, and Smart Loads," IEEE Transactions on Industrial Informatics, vol. 7, no. 3, pp. 381-388, August 2011.

[9] United States Department of Energy, "Smart Grid: an Introduction," Office of Electricity Delivery and Energy Reliability, Washington DC, 2008.

[10] Antonio J. Conejo, Juan M. Morales and Luis Baringo, "RealTime Demand Response Mode," IEEE Transactions on Smart Grid, vol. 1, no. 3, pp. 236-242, December 2010.

[11] Amit Mohan Saklani, H. S. V. S. Kumar Nunna and SuryanarayanaDoolla, "Intelligent Demand Response Approach in Smart Distribution Systems: A Review," in Annual IEEE India Conference, December 2012.

[12] Omid AmeriSianaki, Omar Hussain, Tharam Dillon and AzadehRajabianTabesh, "Intelligent Decision Support System for Including Consumers' Preferences in Residential Energy Consumption in Smart Grid," in Computational Intelligence, Modelling and Simulation, September 2010.

[13] Wang and M. de Groot, "Managing End-User Preferences in the Smart Grid," ACM e-Energy, pp. 105-114, 2010.

[14] KamalanathSamarakoon, JanakaEkanayake and Nick Jenkins, "Reporting Available Demand Response," IEEE Transactions on Smart Grid, 2013.
[15] ThillainathanLogenthiran, Dipti Srinivasan, and Tan Zong Shun, "Demand Side Management in Smart Grid Using Heuristic Optimization," IEEE Transactions on Smart Grid, vol. 3, no. 3, pp. 1244-1252, September 2012.

[16] Yunfei Wang, IrajRahimiPordanjani and Wilsun $\mathrm{Xu}$, "An Event-Driven Demand Response Scheme for Power System Security Enhancement," IEEE Transactions on Smart Grid, vol. 2, no. 1, pp. 23-29, March 2011.

[17] J. Short, D. Infield and L. Freris, "Stabilization of Grid Frequency Through Dynam-ic Demand Control," IEEE Transactions on Power Systems, vol. 22, no. 3, pp. 1284-1293, 2007.

[18] I. Roytelman and V. Ganesan, "Coordinated Local and Centralized Control in Distribution Management Systems," IEEE Transactions on Power Delivery, vol. 15, no. 2, pp. 718724, 2000.

[19] Mohsenian-Rad, V. Wong, J. Jatskevich and R. Schober, "Optimal and Autonomous Incentive-Based Energy Consumption Scheduling Algorithm for Smart Grid," IEEE Innovative Smart Grid Technologies, pp. 1-6, January 2010.

[20] Xiang Lu, Wenye Wang and Jianfeng Ma, "An Empirical Study of Communication Infrastructures Towards the Smart Grid: Design, Implementation, and Evaluation," IEEE Transactions on Smart Grid, vol. 4, no. 1, pp. 170-183, March 2013.

[21] C. Gellings et al., "Integrating Demand-Side Management into Utility Planning," IEEE Transactions on Power Systems, vol. 1, no. 3, pp. 81-87, 1986.

[22] Jose Medina, Nelson Muller and Ilya Roytelman, "Demand Response and Distribution Grid Operations: Opportunities and Challenges," IEEE Transactions on Smart Grid, vol. 1, no. 2, pp. 193-198, September 2010.

[23] C. Su and D. Kirschen, "Quantifying the Effect of Demand Response on Electricity Markets," IEEE Transactions on Power Systems, vol. 24, no. 3, pp. 1199-1207, 2009.

[24] Dan Yang and Yanni Chen, "Demand Response and Market Performance in Power Economics," in IEEE Power and Energy Society General Meeting, July 2009.

[25] "IEEE recommended practice for data communications between remote terminal units and intelligent electronic devices in a substation," IEEE Std 1379-2000, pp. 1-72, 2001. 